16

\title{
О фокусировке поверхностных плазмонных волн на свободной поверхности металлической пленки
}

\author{
(C) А.Б. Петрин \\ Объединенный институт высоких температур РАН, \\ 125412 Москва, Россия \\ e-mail: a_petrin@mail.ru \\ Поступила в редакцию 19.09.2018 г. \\ В окончательной редакции 01.11.18 г. \\ Принята к публикации 06.11.18 г.
}

На основе теории отражения плоской электромагнитной волны от плоскослоистой структуры рассмотрены возбуждение и фокусировка поверхностной плазмонной волны на свободной поверхности металлической пленки в схеме Кречмана. Предложен метод возбуждения радиально сходящейся поверхностной плазмонной волны. Количественно исследовано распределение электрического поля в фокусе и найдены условия его максимизации. Обсуждены приложения полученных результатов.

DOI: $10.21883 /$ OS.2019.03.47378.276-18

\section{Введение и постановка задачи}

В последнее время значительные усилия направлены на развитие методов локального исследования химического состава поверхностей с нанометровым разрешением. Одним из методов является сканирование поверхности нановершиной конического микроострия, на котором сфокусирована сходящаяся поверхностная плазмонная волна оптического диапазона частот [1]. В фокусе (на нановершине) создается настолько сильное оптическое поле, что при попадании в область его действия поверхностной молекулы возникает уникальное для этой молекулы комбинационное излучение. При сканировании исследуемой поверхности оптически возбужденной нановершиной и изучении соответствующего рамановского спектра можно получить химическую карту поверхности с нанометровой точностью. Кроме указанного приложения острийной нанофокусировки к рамановской спектроскопии поверхности, развиваются также и другие приложения, например, связанные с генерацией в сильном оптическом поле фокуса (на нановершине) горячих электронов и оптического излучения при столкновении этих электронов с исследуемой поверхностью (hot electron spectroscopy) [2]. Предлагается использовать указанное явление для поверхностного картирования полупроводниковых наноструктур [3].

Оптическое возбуждение нановершины обычно создают следующим образом. Сначала фокусируют свободно распространяющуюся лазерную волну на устройстве возбуждения поверхностной плазмонной волны, сходящейся к коническому микроострию. В качестве согласующего устройства используют дифракционные решетки $[4,5]$ или фотонные кристаллы [6,7]. Сфокусированная поверхностная плазмонная волна должна создавать на поверхности конического микроострия ТМ-волну, сходящуюся к вершине - фокусу. Следует отметить, что хотя устройства возбуждения нановершины созданы экспериментально [1], вопрос, насколько оптимальны экспериментальные образцы и согласующие устройства, теоретически не решен.

В настоящей работе теоретически рассматривается задача оптимального возбуждения радиально сходящейся поверхностной плазмонной волны, которую можно было бы использовать как самостоятельное устройство, так и для возбуждения металлических микроострий. Решение этой задачи основано на исследовании возбуждения поверхностной плазмонной волны в схеме Кречмана (рис. 1) оптическими пучками ограниченной апертуры [8]. Оказалось (рис. 4, $b$ работы [8]), что ширина падающего пучка в схеме Кречмана должна быть достаточно велика. Это необходимо, чтобы поверхностная волна выросла до максимально возможной амплитуды. В работе [8] было предложено несколько способов увеличения максимального поля поверхностной волны на свободной границе пленки посредством создания нескольких интерферирующих волн, возбужденных каждая своим когерентным пучком. Было показано, что с помощью четырех волн можно создать на свободной поверхности серебряной пленки фокальные пятна размером порядка длины волны поверхностного плазмона и нормированной амплитудой нормальной компоненты электрического поля приблизительно 73.6. Нормировка проводилась на амплитуду падающей волны в призме.

В рамках обычной схемы Кречмана [9] (рис. 1) согласование поверхностной плазмонной волны 1 на поверхности металлической пленки 2 и падающей волны 3 осуществляется с помощью стеклянной призмы 4. Линейно поляризованная плоская монохроматическая волна $\mathbf{E}^{i}$ падает на металлическую пленку со стороны призмы. Вектор напряженности электрического поля падающей волны лежит в плоскости падения ( $p$-поляризация). Призма необходима для согласования падающей плоской волны и поверхностной плазмонной волны по волновому числу. Волновое число поверхностной 


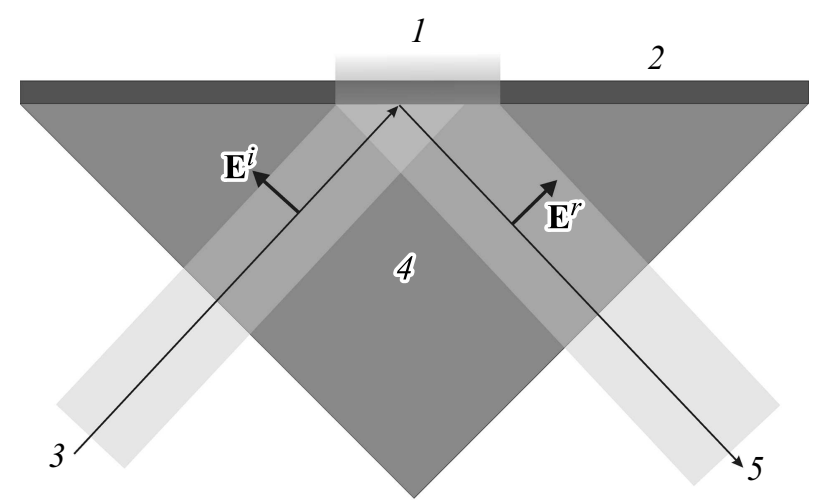

Рис. 1. Возбуждение поверхностных плазмонов 1 на поверхности металлической пленки 2 по схеме Кречмана. Падающая волна 3 в призме 4 порождает поверхностную плазмонную волну 1 и отраженную волну 5 .

плазмонной волны больше волнового числа плоской волны в свободном пространстве той же частоты [10,11]. Чтобы обеспечить равенство волновых чисел падающей 3 , отраженной 5 и поверхностной плазмонной 1 волн вдоль поверхности металла (рис. 1), необходимо, чтобы падающая волна подходила к пленке из оптически более плотной среды (где волновое число больше, чем в свободном пространстве). Показатель преломления призмы и угол падения подбираются таким образом, чтобы обеспечивалось равенство волновых чисел падающей и поверхностных волн. При этих параметрах в отсутствие металлической пленки (и возбуждения поверхностных плазмонов) наблюдается полное внутреннее отражение падающей волны.

Экспериментальные и теоретические исследования показывают, что если угол падения соответствует согласованию по волновому числу падающей и поверхностной плазмонной волн, наблюдается резкое падение коэффициента отражения. Например, при длине падающей волны в вакууме $\lambda=633 \mathrm{~nm}$ и толщине пленки серебра приблизительно равной $53.8 \mathrm{~nm}$ (эта величина зависит от конкретного значения показателя преломления призмы и частоты падающей волны) наблюдается сильное изменение коэффициента отражения практически от единицы до нуля в окрестности угла падения, соответствующего согласованию свободной и поверхностной плазмонной волн. При небольшом отклонении угла падения от угла согласования коэффициент отражения возвращается от нуля к единичному значению.

Так как поле поверхностной плазмонной волны сосредоточено в тонком слое вблизи поверхности металла, характер отражения в схеме Кречмана сильно зависит от изменений показателя преломления в тонком слое вблизи поверхности. Именно на этом основывается широкое применение схемы Кречмана для создания различного рода датчиков, обладающих высокой чувствительностью [12].
Возникновение минимума отражения при возбуждении поверхностной волны сопровождается максимумом амплитуды поверхностной волны на внешней границе пленочной структуры с возбужденной поверхностной волной. Эта амплитуда намного больше амплитуды падающей волны. Так, было показано, что для случая возбуждения поверхностной плазмонной волны на поверхности серебряной пленки $[8,13]$ максимальная амплитуда нормальной составляющей электрического поля в 18.78 раз больше амплитуды падающей волны в призме.

В настоящей работе рассматривается логическое продолжение работы [8] - задача точечной фокусировки посредством создания радиально сходящейся поверхностной волны, возбужденной специальной конической волной. Поверхностная плазмонная стоячая волна 1 (рис. 2) возбуждается на поверхности серебряной пленки 2 с помощью конической падающей волны 3 в конической призме 4. Падающая 3 и отраженная 5 конические волны имеют $p$-поляризацию, т.е. вектор напряженности электрического поля лежит в плоскости, проходящей через ось $Z$. В данном исследовании мы будем интересоваться распределением поля сфокусированной волны на свободной поверхности металлической пленки 2 и значением амплитуды напряженности поля в фокусе.

\section{Общая задача падения электромагнитной волны на плоскослоистую структуру}

Рассмотрим задачу падения монохроматической волны с циклической частотой $\omega$ единичной амплитуды из полупространства (призмы), заполненного однородным изотропным диэлектриком с показателем преломления $n_{1}$, на плоскую пленку толщиной $d_{1}$. Предполагается для определенности, что пленка металлическая с комплексной диэлектрической проницаемостью $\varepsilon_{2}$ на

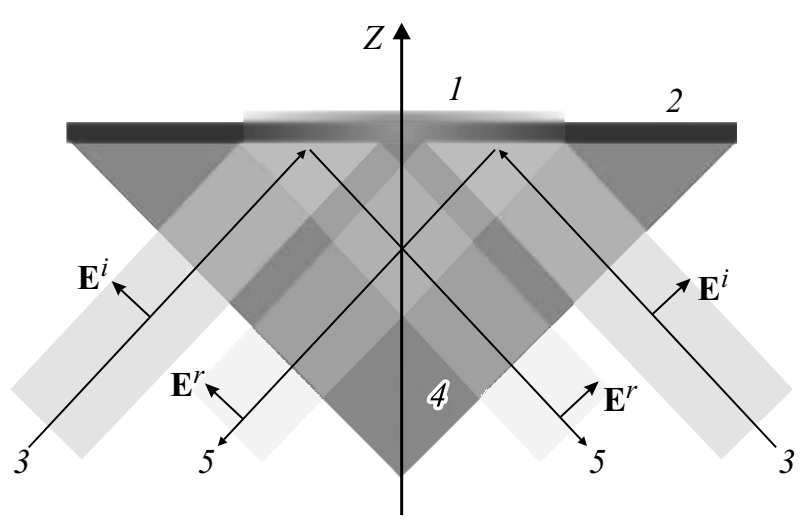

Рис. 2. Возбуждение поверхностной плазмонной стоячей волны 1 на поверхности серебряной пленки 2 с помощью конической волны 3 , падающей со стороны конической призмы 4. Падающая 3 и отраженная 5 конические волны имеют оляризацию. 
рассматриваемой частоте $\omega$, а за пленкой находится однородное полупространство с диэлектрической проницаемостью $\varepsilon_{3}$ (свободное пространство). Сначала будет рассмотрена задача с одной пленкой, а затем она будет обобщена на многопленочную структуру с произвольным количеством пленок. Данный подход к решению электромагнитной задачи для многопленочной структуры восходит к работе А. Зоммерфельда [14] и развит в работах $[15,16]$.

Уравнения Максвелла в рассматриваемых областях можно записать в виде

$$
\begin{gathered}
\operatorname{rot} \mathbf{E}_{j}=i \omega \mathbf{B}_{j}, \\
\operatorname{rot} \mathbf{B}_{j}=-i \omega \varepsilon_{j} \mu_{j} \mathbf{E}_{j},
\end{gathered}
$$

где $\mathbf{E}_{j}$ и $\mathbf{B}_{j}$ - векторы напряженности электрического поля и индукция магнитного поля в области с номером $j ; j=1$ соответствует полупространству 1 , для которого $z<0 ; j=2$ соответствует области пленки 2 $\left(0<z<d_{1}\right) ; j=3$ соответствует свободному полупространству $\left(z>d_{1}\right)$. Комплексное представление во времени предполагается в виде $e^{-i \omega t}$.

Из уравнений (1) и (2) следует, что электрическое и магнитное поля удовлетворяют однородному волновому уравнению, т. е.

$$
\begin{aligned}
& \operatorname{rot} \operatorname{rot} \mathbf{E}_{j}-\omega^{2} \mu_{j} \varepsilon_{j} \mathbf{E}_{j}=0, \\
& \operatorname{rot} \operatorname{rot} \mathbf{B}_{j}-\omega^{2} \mu_{j} \varepsilon_{j} \mathbf{B}_{j}=0
\end{aligned}
$$

Учитывая, что $\operatorname{div} \mathbf{D}_{j}=0 \quad$ или $\partial_{x} E_{j, x}+\partial_{y} E_{j, y}+$ $+\partial_{z} E_{j, z}=0$, из (3) получаем уравнение для $E_{j, x}$ :

$$
\partial_{x x}^{2} E_{j, x}+\partial_{y y}^{2} E_{j, x}+\partial^{2} E_{j, x}+\omega^{2} \mu_{j} \varepsilon_{j} E_{j, x}=0 .
$$

Аналогичные уравнения получаются для компонент напряженности электрического поля $E_{j, y}, E_{j, z}$ и индукции магнитного поля $B_{j, x}, B_{j, y}$ и $B_{j, z}$ (для краткости уравнения не выписываем).

Представим в (4) компоненты напряженности электрических полей в виде фурье-разложений:

$$
\begin{aligned}
& E_{j, x}(x, y, z)=\frac{1}{\left(2 \pi^{2}\right)} \int_{-\infty}^{+\infty} \int_{-\infty}^{+\infty} \tilde{E}_{j, x}(\xi, \eta, z) e^{i(\xi x+\eta y)} d \xi d \eta \\
& E_{j, y}(x, y, z)=\frac{1}{(2 \pi)^{2}} \int_{-\infty}^{+\infty} \int_{-\infty}^{+\infty} \tilde{E}_{j, y}(\xi, \eta, z) e^{i(\xi x+\eta y)} d \xi d \eta \\
& E_{j, z}(x, y, z)=\frac{1}{(2 \pi)^{2}} \int_{-\infty}^{+\infty} \int_{-\infty}^{+\infty} \tilde{E}_{j, z}(\xi, \eta, z) e^{i(\xi x+\eta y)} d \xi d \eta
\end{aligned}
$$

где фурье-образы определяются интегралами

$$
\tilde{E}_{j, x}(\xi, \eta, z)=\int_{-\infty}^{+\infty} \int_{-\infty}^{+\infty} E_{j, x}(x, y, z) e^{-i(\xi x+\eta y)} d x d y,
$$

$$
\begin{aligned}
\tilde{E}_{j, y}(\xi, \eta, z) & =\int_{-\infty}^{+\infty} \int_{-\infty}^{+\infty} E_{j, y}(x, y, z) e^{-i(\xi x+\eta y)} d x d y, \\
\tilde{E}_{j, z}(\xi, \eta, z) & =\int_{-\infty}^{+\infty} \int_{-\infty}^{+\infty} E_{j, z}(x, y, z) e^{-i(\xi x+\eta y)} d x d y .
\end{aligned}
$$

Тогда для фурье-образов полей получим следующее уравнение для $\tilde{E}_{j, x}$ :

$$
\frac{d^{2} E_{j, x}}{d z^{2}}+\left(\omega^{2} \mu_{j} \varepsilon_{j}-\xi^{2}-\eta^{2}\right) \tilde{E}_{j, x}=0
$$

и аналогичные уравнения для $\tilde{E}_{j, y}$ и $\tilde{E}_{j, z}$ (для краткости не выписываем).

Решением этих уравнений являются $\tilde{E}_{j, x}^{ \pm}=\hat{E}_{j, x}^{ \pm} e^{ \pm i k_{j, z} z}$, $\tilde{E}_{j, y}^{ \pm}=\hat{E}_{j, y}^{ \pm} e^{ \pm i k_{j, z} z}, \tilde{E}_{j, z}^{ \pm}=\hat{E}_{j, z}^{ \pm} e^{ \pm i k_{j, z} z}$, где $\hat{E}_{j, x}^{+}, \hat{E}_{j, x}^{-}, \hat{E}_{j, y}^{+}$, $\hat{E}_{j, y}^{-}, \quad \hat{E}_{j, z}^{+}, \quad \hat{E}_{j, z}^{-}-$функции только от $\xi$ и $\eta$; $k_{j, z}=k_{j, z}(\xi, \eta)=\sqrt{\omega^{2} \mu_{j} \varepsilon_{j}-\xi^{2}-\eta^{2}}$.

Тогда общее решение уравнений (5) для направлений распространения волн вдоль „+““ и против „-“ оси $Z$ можно записать в виде

$$
\begin{aligned}
\mathbf{E}_{j}^{ \pm}(x, y, z)= & \frac{1}{(2 \pi)^{2}} \int_{-\infty}^{+\infty} \int_{-\infty}^{+\infty}\left(\begin{array}{c}
\hat{E}_{j, x}^{ \pm}(\xi, \eta) \\
\hat{E}_{j, y}^{ \pm}(\xi, \eta) \\
\hat{E}_{j, z}^{ \pm}(\xi, \eta)
\end{array}\right) e^{ \pm i k_{j, z} z} \\
& \times e^{i(\xi x+\eta y)} d \xi d \eta .
\end{aligned}
$$

Так как $\quad \operatorname{div} \mathbf{D}_{j}=0, \quad$ то $\quad \varepsilon_{j} \xi \hat{E}_{j, x}+\varepsilon_{j} \eta \hat{E}_{j, y} \pm$ $\pm \varepsilon_{j} k_{j, z}(\xi) \hat{E}_{j, z}=0$, и общее решение (6) можно переписать в виде

$$
\begin{aligned}
\mathbf{E}_{j}^{ \pm}(x, y, z)= & \frac{1}{(2 \pi)^{2}} \int_{-\infty}^{+\infty} \int_{-\infty}^{+\infty}\left(\begin{array}{cc}
1 & 0 \\
0 & 1 \\
\mp \frac{\xi}{k_{j, z}} & \mp \frac{\eta}{k_{j, z}}
\end{array}\right) \\
& \times\left(\begin{array}{c}
\hat{E}_{j, x}^{ \pm}(\xi, \eta) \\
\hat{E}_{j, y}^{ \pm}(\xi, \eta)
\end{array}\right) e^{ \pm i k_{j, z} z} e^{i(\xi x+\eta y)} d \xi d \eta .
\end{aligned}
$$

Запишем общее решение в рассматриваемых областях (рис. 2) в следующем виде:

в области 1

$$
\begin{aligned}
\mathbf{E}_{1}(x, y, z)= & \frac{1}{(2 \pi)^{2}} \int_{-\infty}^{+\infty} \int_{-\infty}^{+\infty}\left(\begin{array}{cc}
1 & 0 \\
0 & 1 \\
-\frac{\xi}{k_{1, z}} & -\frac{\eta}{k_{1, z}}
\end{array}\right)\left(\begin{array}{c}
\hat{E}_{1, x}^{+} \\
\hat{E}_{1, y}^{+}
\end{array}\right) \\
& \times e^{i k_{1, z} z} e^{i(\xi x+\eta y)} d \xi d \eta \\
& +\frac{1}{(2 \pi)^{2}} \int_{-\infty}^{+\infty} \int_{-\infty}^{+\infty}\left(\begin{array}{cc}
1 & 0 \\
0 & 1 \\
\frac{\xi}{k_{1, z}} & \frac{\eta}{k_{1, z}}
\end{array}\right)\left(\begin{array}{c}
\hat{E}_{1, x}^{-} \\
\hat{E}_{1, y}^{-}
\end{array}\right) \\
& \times e^{-i k_{1, z} z} e^{i(\xi x+\eta y)} d \xi d \eta,
\end{aligned}
$$


в области 2

$$
\begin{aligned}
\mathbf{E}_{2}(x, y, z)= & \frac{1}{(2 \pi)^{2}} \int_{-\infty}^{+\infty} \int_{-\infty}^{+\infty}\left(\begin{array}{cc}
1 & 0 \\
0 & 1 \\
-\frac{\xi}{k_{2, z}} & -\frac{\eta}{k_{2, z}}
\end{array}\right)\left(\begin{array}{l}
\hat{E}_{2, x}^{+} \\
\hat{E}_{2, y}^{+}
\end{array}\right) \\
& \times e^{i k_{2, z} z} e^{i(\xi x+\eta y)} d \xi d \eta \\
& +\frac{1}{(2 \pi)^{2}} \int_{-\infty}^{+\infty} \int_{-\infty}^{+\infty}\left(\begin{array}{cc}
1 & 0 \\
0 & 1 \\
\frac{\xi}{k_{2, z}} & \frac{\eta}{k_{2, z}}
\end{array}\right)\left(\begin{array}{l}
\hat{E}_{2, x}^{-} \\
\hat{E}_{2, y}^{-}
\end{array}\right) \\
& \times e^{-i k_{2, z}\left(z-d_{1}\right)} e^{i(\xi x+\eta y)} d \xi d \eta,
\end{aligned}
$$

в области 3

$$
\begin{aligned}
\mathbf{E}_{3}(x, y, z)= & \frac{1}{(2 \pi)^{2}} \int_{-\infty}^{+\infty} \int_{-\infty}^{+\infty}\left(\begin{array}{cc}
1 & 0 \\
0 & 1 \\
-\frac{\xi}{k_{3, z}} & -\frac{\eta}{k_{3, z}}
\end{array}\right)\left(\begin{array}{l}
\hat{E}_{3, x}^{+} \\
\hat{E}_{3, y}^{+}
\end{array}\right) \\
& \times e^{i k_{3, z}\left(z-d_{1}\right)} e^{i(\xi x+\eta y)} d \xi d \eta
\end{aligned}
$$

Аналогичные выражения в трех областях можно написать и для индукции магнитного поля.

Из уравнения $\operatorname{rot} \mathbf{E}_{j}=i \omega \mathbf{B}_{j}$ найдем $x$ - и $y$-компоненты индукции магнитного поля. Для соответствующих фурье-компонент найдем

$$
\begin{aligned}
\hat{\mathbf{B}}_{j}^{+}= & \frac{1}{i \omega}\left|\begin{array}{ccc}
\mathbf{e}_{x} & \mathbf{e}_{y} & \mathbf{e}_{z} \\
i \xi & i \eta & i k_{j, z} \\
\hat{E}_{j, x}^{+} & \hat{E}_{j, y}^{+} & \hat{E}_{j, z}^{+}
\end{array}\right|=\mathbf{e}_{x}\left(\frac{\eta}{\omega} \hat{E}_{j, z}^{+}-\frac{k_{j, z}}{\omega} \hat{E}_{j, y}^{+}\right) \\
& -\mathbf{e}_{y}\left(\frac{\xi}{\omega} \hat{E}_{j, z}^{+}-\frac{k_{j, z}}{\omega} \hat{E}_{j, x}^{+}\right)+\mathbf{e}_{z}\left(\frac{\xi}{\omega} \hat{E}_{j, y}^{+}-\frac{\eta}{\omega} \hat{E}_{j, x}^{+}\right),
\end{aligned}
$$

или, учитывая, что $\hat{E}_{j, z}^{+}=-\frac{\xi}{k_{j, x}}-\frac{\eta}{k_{j, z}} \hat{E}_{j, y}^{+}$,

$$
\begin{aligned}
\hat{\mathbf{B}}_{j}^{+}= & \mathbf{e}_{x}\left(-\frac{\xi \eta}{\omega k_{j, z}} \hat{E}_{j, x}-\left(\frac{k_{j, z}^{2}+\eta^{2}}{\omega k_{j, z}}\right) \hat{E}_{j, y}^{+}\right) \\
& +\mathbf{e}_{y}\left(\left(\frac{k_{j, z}^{2}+\xi^{2}}{\omega k_{j, z}}\right) \hat{E}_{j, x}^{+}+\frac{\xi \eta}{\omega k_{j, z}} \hat{E}_{j, y}^{+}\right) \\
& +\mathbf{e}_{z}\left(\frac{\xi}{\omega} \hat{E}_{j, y}^{+}-\frac{\eta}{\omega} \hat{E}_{j, x}^{+}\right),
\end{aligned}
$$

или в матричном виде

$$
\left(\begin{array}{c}
\hat{B}_{j, x}^{+} \\
\hat{B}_{j, y}^{+} \\
\hat{B}_{j, z}^{+}
\end{array}\right)=\left[\begin{array}{cc}
-\frac{\xi \eta}{\omega k_{j, z}} & -\left(\frac{k_{j, z}^{2}+\eta^{2}}{\omega k_{j, z}}\right) \\
\left(\frac{k_{j, z}^{2} \xi^{2}}{\omega k_{j, z}}\right) & \frac{\xi \eta}{\omega k_{j, z}} \\
-\frac{\eta}{\omega} & \frac{\xi}{\omega}
\end{array}\right]\left(\begin{array}{c}
\hat{E}_{j, x}^{+} \\
\hat{E}_{j, y}^{+}
\end{array}\right) .
$$

Аналогично для $\hat{\mathbf{B}}_{j}^{-}$найдем

$$
\left(\begin{array}{l}
\hat{B}_{j, x}^{-} \\
\hat{B}_{j, y}^{-} \\
\hat{B}_{j, z}^{-}
\end{array}\right)=\left[\begin{array}{cc}
\frac{\xi \eta}{\omega k_{j, z}} & \left(\frac{k_{j, z}^{2}+\eta^{2}}{\omega k_{j, z}}\right) \\
-\left(\frac{k_{j, z}^{2}+\xi^{2}}{\omega k_{j, z}}\right) & -\frac{\xi \eta}{\omega k_{j, z}} \\
-\frac{\eta}{\omega} & \frac{\xi}{\omega}
\end{array}\right]\left(\begin{array}{l}
\hat{E}_{j, x}^{-} \\
\hat{E}_{j, y}^{-}
\end{array}\right) .
$$

Тогда для тангенциальных компонент можно записать следующие матричные соотношения:

$$
\begin{gathered}
\left(\begin{array}{c}
\hat{B}_{j, x}^{+} \\
\hat{B}_{j, y}^{+}
\end{array}\right)=\left[\begin{array}{cc}
-\frac{\xi \eta}{\omega k_{j, z}} & -\frac{k_{j, z}^{2}+\eta^{2}}{\omega k_{j, z}} \\
\frac{k_{j, z}^{2}+\xi^{2}}{\omega k_{j, z}} & \frac{\xi \eta}{\omega k_{j, z}}
\end{array}\right]\left(\begin{array}{l}
\hat{E}_{j, x}^{+} \\
\hat{E}_{j, y}^{+}
\end{array}\right), \\
\left(\begin{array}{l}
\hat{B}_{j, x}^{-} \\
\hat{B}_{j, y}^{-}
\end{array}\right)=\left[\begin{array}{cc}
\frac{\xi \eta}{\omega k_{j, z}} & \frac{k_{j, z}^{2}+\eta^{2}}{\omega k_{j, z}} \\
-\frac{k_{j, z}^{2}+\xi^{2}}{\omega k_{j, z}} & -\frac{\xi \eta}{\omega k_{j, z}}
\end{array}\right]\left(\begin{array}{l}
\hat{E}_{j, x}^{-} \\
\hat{E}_{j, y}^{-}
\end{array}\right),
\end{gathered}
$$

Прежде чем записать граничные условия, выпишем тангенциальные компоненты напряженности электрического поля и индукции магнитного поля:

в области 1

$$
\begin{aligned}
& \mathbf{E}_{1, \tau}= \frac{1}{(2 \pi)^{2}} \int_{-\infty}^{+\infty} \int_{-\infty}^{+\infty}\left(\begin{array}{ll}
1 & 0 \\
0 & 1
\end{array}\right)\left(\begin{array}{l}
\hat{E}_{1, x}^{+} \\
\hat{E}_{1, y}^{+}
\end{array}\right) e^{i k_{1, z} z} e^{i(\xi x+\eta y)} d \xi d \eta \\
&+\frac{1}{(2 \pi)^{2}} \int_{-\infty}^{+\infty} \int_{-\infty}^{+\infty}\left(\begin{array}{ll}
1 & 0 \\
0 & 1
\end{array}\right)\left(\begin{array}{l}
\hat{E}_{1, x}^{-} \\
\hat{E}_{1, y}^{-}
\end{array}\right) e^{-k_{1, z} z} e^{i(\xi x+\eta y)} d \xi d \eta \\
& \mathbf{B}_{1, \tau}= \frac{1}{(2 \pi)^{2}} \int_{-\infty}^{+\infty} \int_{-\infty}^{+\infty}\left(\begin{array}{ll}
-\frac{\xi \eta}{\omega k_{1, z}} & -\frac{k_{1,2}^{2}+\eta^{2}}{\omega k_{1, z}} \\
\frac{k_{1, z}^{2}+\xi^{2}}{\omega k_{1, z}} & \frac{\xi \eta}{\omega k_{1, z}}
\end{array}\right)\left(\begin{array}{l}
\hat{E}_{1, x}^{+} \\
\hat{E}_{1, y}^{+}
\end{array}\right) \\
& \times e^{i k_{1, z} z} e^{i(\xi x+\eta y) d \xi d \eta} \\
&+\frac{1}{(2 \pi)^{2}} \int_{-\infty}^{+\infty} \int_{-\infty}^{+\infty}\left(\begin{array}{cc}
\frac{\xi \eta}{\omega k_{1, z}} & \frac{k_{1, z}^{2}+\eta^{2}}{\omega k_{1, z}} \\
-\frac{k_{1, z}^{2}+\xi^{2}}{\omega k_{1, z}} & -\frac{\xi \eta}{\omega k_{1, z}}
\end{array}\right)\left(\begin{array}{l}
\hat{E}_{1, x}^{-} \\
\hat{E}_{1, y}^{-}
\end{array}\right) \\
& \times e^{-i k_{1, z} z} e^{i(\xi x+\eta y)} d \xi d \eta,
\end{aligned}
$$

в области 2

$$
\begin{gathered}
\mathbf{E}_{2, \tau}=\frac{1}{(2 \pi)^{2}} \int_{-\infty}^{+\infty} \int_{-\infty}^{+\infty}\left(\begin{array}{ll}
1 & 0 \\
0 & 1
\end{array}\right)\left(\begin{array}{l}
\hat{E}_{2, x}^{+} \\
\hat{E}_{2, y}^{+}
\end{array}\right) e^{i k_{2, z} z} e^{i(\xi x+\eta y)} d \xi d \eta \\
+\frac{1}{(2 \pi)^{2}} \int_{-\infty}^{+\infty} \int_{-\infty}^{+\infty}\left(\begin{array}{ll}
1 & 0 \\
0 & 1
\end{array}\right)\left(\begin{array}{c}
\hat{E}_{2, x}^{-} \\
\hat{E}_{2, y}^{-}
\end{array}\right) e^{-i k_{2, z}\left(z-d_{1}\right)} e^{i(\xi x+\eta y)} d \xi d \eta
\end{gathered}
$$

$$
\begin{aligned}
\mathbf{B}_{2, \tau}= & \frac{1}{(2 \pi)^{2}} \int_{-\infty}^{+\infty} \int_{-\infty}^{+\infty}\left(\begin{array}{cc}
-\frac{\xi \eta}{\omega k_{2, z}} & -\frac{k_{2, z}^{2}+\eta^{2}}{\omega k_{2, z}} \\
\frac{k_{1,2}^{2}+\xi^{2}}{\omega k_{2, z}} & \frac{\xi \eta}{\omega k_{2, z}}
\end{array}\right)\left(\begin{array}{c}
\hat{E}_{2, x}^{+} \\
\hat{E}_{2, y}^{+}
\end{array}\right) \\
& \times e^{i k_{2, z} z} e^{i(\xi x+\eta y)} d \xi d \eta \\
& +\frac{1}{(2 \pi)^{2}} \int_{-\infty}^{+\infty} \int_{-\infty}^{+\infty}\left(\begin{array}{cc}
\frac{\xi \eta}{\omega k_{2, z}} & \frac{k_{2, z}^{2}+\eta^{2}}{\omega k_{2, z}} \\
-\frac{k_{2,2}^{2}+\xi^{2}}{\omega k_{2, z}} & -\frac{\xi \eta}{\omega k_{2, z}}
\end{array}\right)\left(\begin{array}{l}
\hat{E}_{2, x}^{-} \\
\hat{E}_{2, y}^{-}
\end{array}\right) \\
& \times e^{-i k_{1, z}\left(z-d_{1}\right)} e^{i(\xi x+\eta y)} d \xi d \eta,
\end{aligned}
$$


в области 3

$$
\begin{gathered}
\mathbf{E}_{3, \tau}=\frac{1}{(2 \pi)^{2}} \int_{-\infty}^{+\infty} \int_{-\infty}^{+\infty}\left(\begin{array}{ll}
1 & 0 \\
0 & 1
\end{array}\right)\left(\begin{array}{c}
\hat{E}_{3, x}^{+} \\
\hat{E}_{3, y}^{+}
\end{array}\right) \\
\times e^{i k_{3, z}\left(z-d_{1}\right)} e^{i(\xi x+\eta y)} d \xi d \eta \\
\mathbf{B}_{3, \tau}=\frac{1}{(2 \pi)^{2}} \int_{-\infty}^{+\infty} \int_{-\infty}^{+\infty}\left(\begin{array}{cc}
-\frac{\xi \eta}{\omega k_{3, z}} & -\frac{k_{3, z}^{2}+\eta^{2}}{\omega k_{3, z}} \\
\frac{k_{3,2}^{2}+\xi^{2}}{\omega k_{3, z}} & \frac{\xi \eta}{\omega k_{3, z}}
\end{array}\right)\left(\begin{array}{c}
\hat{E}_{3, x}^{+} \\
\hat{E}_{3, y}^{+}
\end{array}\right) \\
\times e^{i k_{3, z}\left(z-d_{1}\right)} e^{i(\xi x+\eta y)} d \xi d \eta .
\end{gathered}
$$

Полученные выражения для полей в рассматриваемых областях должны удовлетворять граничным условиям. Известно, что для гармонических полей на границах должны быть непрерывны тангенциальные компоненты напряженности электрического поля и индукции магнитного поля.

Рассмотрим границу первой и второй областей $(z=0)$. На ней непрерывность тангенциальных компонент можно записать в виде

$E_{1, x}(x, y, 0)=E_{2, x}(x, y, 0), \quad E_{1, y}(x, y, 0)=E_{2, y}(x, y, 0)$,

$$
\begin{aligned}
& B_{1, x}(x, y, 0) / \mu_{1}=B_{2, x}(x, y, 0) / \mu_{2}, \\
& B_{1, y}(x, y, 0) / \mu_{1}=B_{2, y}(x, y, 0) / \mu_{2} .
\end{aligned}
$$

Учитывая, что

$$
\begin{aligned}
& E_{1, x}(x, y, 0)=E_{1, x}^{+}(x, y, 0)+E_{1, x}^{-}(x, y, 0), \\
& E_{1, y}(x, y, 0)=E_{1, y}^{+}(x, y, 0)+E_{1, y}^{-}(x, y, 0), \\
& E_{2, x}(x, y, 0)=E_{2, x}^{+}(x, y, 0)+E_{2, x}^{-}(x, y, 0), \\
& E_{2, y}(x, y, 0)=E_{2, y}^{+}(x, y, 0)+E_{2, y}^{-}(x, y, 0), \\
& B_{1, x}(x, y, 0)=B_{1, x}^{+}(x, y, 0)+B_{1, x}^{-}(x, y, 0), \\
& B_{1, y}(x, y, 0)=B_{1, y}^{+}(x, y, 0)+B_{1, y}^{-}(x, y, 0), \\
& B_{2, x}(x, y, 0)=B_{2, x}^{+}(x, y, 0)+B_{2, x}^{-}(x, y, 0), \\
& B_{2, y}(x, y, 0)=B_{2, y}^{+}(x, y, 0)+B_{2, y}^{-}(x, y, 0),
\end{aligned}
$$

после несложных преобразований, используя (13)-(16), из граничных условий получим следующие матричные уравнения:

$$
\left(\begin{array}{cc}
\mathbf{I} & \mathbf{I} \\
\mathbf{G}_{1}^{+} & \mathbf{G}_{1}^{-}
\end{array}\right)\left(\begin{array}{l}
\hat{E}_{1, x}^{+} \\
\hat{E}_{1, y}^{+} \\
\hat{E}_{1, x}^{-} \\
\hat{E}_{1, y}^{-}
\end{array}\right)\left(\begin{array}{cc}
\mathbf{I} & e^{i k_{2, z} d_{1}} \mathbf{I} \\
\mathbf{G}_{2}^{+} & e^{i k_{2, z} d_{1}} \mathbf{G}_{2}^{-}
\end{array}\right)\left(\begin{array}{c}
\hat{E}_{2, x}^{+} \\
\hat{E}_{2, y}^{+} \\
\hat{E}_{2, x}^{-} \\
\hat{E}_{2, y}^{-}
\end{array}\right)
$$

или

$$
\left(\begin{array}{c}
\hat{E}_{1, x}^{+} \\
\hat{E}_{1, y}^{+} \\
\hat{E}_{1, x}^{-} \\
\hat{E}_{1, y}^{-,}
\end{array}\right)=\left(\begin{array}{cc}
\mathbf{I} & \mathbf{I} \\
\mathbf{G}_{1}^{+} & \mathbf{G}_{1}^{-}
\end{array}\right)^{-1}\left(\begin{array}{cc}
\mathbf{I} & e^{i k_{2, z} d_{1}} \mathbf{I} \\
\mathbf{G}_{2}^{+} & e^{i k_{2, z} d_{1}} \mathbf{G}_{2}^{-}
\end{array}\right)\left(\begin{array}{l}
\hat{E}_{2, x}^{+} \\
\hat{E}_{2, y}^{+} \\
\hat{E}_{2, x}^{-} \\
\hat{E}_{2, y}^{-}
\end{array}\right),
$$

где I - единичная матрица $2 \times 2$, а остальные матрицы имеют вид

$\mathbf{G}_{1}^{+}=\left(\begin{array}{cc}-\frac{\xi \eta}{\omega \mu_{1} k_{1, z}} & -\frac{k_{1, z}^{2}+\eta^{2}}{\omega \mu_{1} k_{1, z}} \\ \frac{k_{1, z}^{2}+\xi^{2}}{\omega \mu_{1} k_{1, z}} & \frac{\xi \eta}{\omega \mu_{1} k_{1, z}}\end{array}\right), \quad \mathbf{G}_{1}^{-}=\left(\begin{array}{cc}\frac{\xi \eta}{\omega \mu_{1} k_{1, z}} & \frac{k_{1, z}^{2}+\eta^{2}}{\omega \mu_{1} k_{1, z}} \\ -\frac{k_{1, z}^{2}+\xi^{2}}{\omega \mu_{1} k_{1, z}} & -\frac{\xi \eta}{\omega \mu_{1} k_{1, z}}\end{array}\right)$,

$\mathbf{G}_{2}^{+}=\left(\begin{array}{cc}-\frac{\xi \eta}{\omega \mu_{2} k_{2, z}} & -\frac{k_{2, z}^{2}+\eta^{2}}{\omega \mu_{2} k_{2, z}} \\ \frac{k_{2, z}^{2}+\xi^{2}}{\omega \mu_{2} k_{2, z}} & \frac{\xi \eta}{\omega \mu_{2} k_{2, z}}\end{array}\right), \quad \mathbf{G}_{2}^{-}=\left(\begin{array}{cc}\frac{\xi \eta}{\omega \mu_{2} k_{2, z}} & \frac{k_{2, z}^{2}+\eta^{2}}{\omega \mu_{2} k_{2, z}} \\ -\frac{k_{2, z}^{2}+\xi^{2}}{\omega \mu_{2} k_{2, z}} & -\frac{\xi \eta}{\omega \mu_{2} k_{2, z}}\end{array}\right)$.

Аналогично рассмотрим границу второй и третьей областей $\left(z=d_{1}\right)$. Граничные условия запишутся в следующем виде:

$$
\begin{aligned}
E_{2, x}\left(x, y, d_{1}\right) & =E_{3, x}\left(x, y, d_{1}\right), \\
E_{2, y}\left(x, y, d_{1}\right) & =E_{3, y}\left(x, y, d_{1}\right), \\
B_{2, x}\left(x, y, d_{1}\right) / \mu_{2} & =B_{3, x}\left(x, y, d_{1}\right) / \mu_{3}, \\
B_{2, y}\left(x, y, d_{1}\right) / \mu_{2} & =B_{3, y}\left(x, y, d_{1}\right) / \mu_{3} .
\end{aligned}
$$

Используя выражения (15)-(18), получим следующие матричные уравнения:

$$
\left(\begin{array}{cc}
e^{i k_{2, z} d_{1}} \mathbf{I} & \mathbf{I} \\
e^{i k_{2, z} d_{1}} \mathbf{G}_{2}^{+} & \mathbf{G}_{2}^{-}
\end{array}\right)\left(\begin{array}{c}
\hat{E}_{2, x}^{+} \\
\hat{E}_{2, y}^{+} \\
\hat{E}_{2, x}^{-} \\
\hat{E}_{2, y}^{-}
\end{array}\right)=\left(\begin{array}{cc}
\mathbf{I} & 0 \\
\mathbf{G}_{3}^{+} & 0
\end{array}\right)\left(\begin{array}{c}
\hat{E}_{3, x}^{+} \\
\hat{E}_{3, y}^{+} \\
0 \\
0
\end{array}\right)
$$

или

$$
\left(\begin{array}{c}
\hat{E}_{2, x}^{+} \\
\hat{E}_{2, y}^{+} \\
\hat{E}_{2, x}^{-} \\
\hat{E}_{2, y}^{-}
\end{array}\right)=\left(\begin{array}{cc}
e^{i k_{2, z} d_{1}} \mathbf{I} & \mathbf{I} \\
e^{i k_{2, z} d_{1}} \mathbf{G}_{2}^{+} & \mathbf{G}_{\mathbf{2}}^{-}
\end{array}\right)\left(\begin{array}{cc}
\mathbf{I} & 0 \\
\mathbf{G}_{3}^{+} & 0
\end{array}\right)\left(\begin{array}{c}
\hat{E}_{3, x}^{+} \\
\hat{E}_{3, y}^{+} \\
0 \\
0
\end{array}\right),
$$

где

$$
\mathbf{G}_{3}^{+}=\left(\begin{array}{cc}
-\frac{\xi \eta}{\omega \mu_{3} k_{3, z}} & -\frac{k_{3, z}^{2}+\eta^{2}}{\omega \mu_{3} k_{3, z}} \\
\frac{k_{3, z}^{2}+\xi^{2}}{\omega \mu_{3} k_{3, z}} & \frac{\xi \eta}{\omega \mu_{3} k_{3, z}}
\end{array}\right)
$$


Из матричных уравнений (19),(20) получаем

$$
\begin{aligned}
& \left(\begin{array}{c}
\hat{E}_{1, x}^{+} \\
\hat{E}_{1, y}^{+} \\
\hat{E}_{1, x}^{-} \\
\hat{E}_{1, y}^{-}
\end{array}\right)=\left(\begin{array}{cc}
\mathbf{I} & \mathbf{I} \\
\mathbf{G}_{1}^{+} & \mathbf{G}_{1}^{-}
\end{array}\right)^{-1}\left(\begin{array}{cc}
\mathbf{I} & e^{i k_{2, z} d_{1} \mathbf{I}} \\
\mathbf{G}_{2}^{+} & e^{i k_{2, z} d_{1}} \mathbf{G}_{2}^{-}
\end{array}\right) \\
& \times\left(\begin{array}{cc}
e^{i k_{2, z} d_{1} \mathbf{I}} & \mathbf{I} \\
e^{i k_{2, z} d_{1}} \mathbf{G}_{2}^{+} & \mathbf{G}_{2}^{-}
\end{array}\right)^{-1}\left(\begin{array}{cc}
\mathbf{I} & 0 \\
\mathbf{G}_{3}^{+} & 0
\end{array}\right)\left(\begin{array}{c}
\hat{E}_{3, x}^{+} \\
\hat{E}_{x, y}^{+} \\
0 \\
0
\end{array}\right)
\end{aligned}
$$

или

$$
\left(\begin{array}{c}
\hat{E}_{1, x}^{+} \\
\hat{E}_{1, y}^{+} \\
\hat{E}_{1, x}^{-} \\
\hat{E}_{1, y}^{-}
\end{array}\right)=\mathbf{M}\left(\begin{array}{c}
\hat{E}_{3, x}^{+} \\
\hat{E}_{3, y}^{+} \\
0 \\
0
\end{array}\right),
$$

где матрица М представляется в виде произведения трех матриц $\mathbf{M}=\mathbf{T}_{1} \mathbf{T}_{2} \mathbf{T}_{3}$, в котором

$$
\begin{gathered}
\mathbf{T}_{1}=\left(\begin{array}{cc}
\mathbf{I} & \mathbf{I} \\
\mathbf{G}_{1}^{+} & \mathbf{G}_{1}^{-}
\end{array}\right)^{-1}, \\
\mathbf{T}_{2}=\left(\begin{array}{cc}
\mathbf{I} & e^{i k_{2, z} d_{1} \mathbf{I}} \\
\mathbf{G}_{2}^{+} & e^{i k_{2, z} d_{1}} \mathbf{G}_{2}^{-}
\end{array}\right)\left(\begin{array}{cc}
e^{i k_{2, z} d_{1} \mathbf{I}} & \mathbf{I} \\
e^{i k_{2, z} d_{1}} \mathbf{G}_{2}^{+} & \mathbf{G}_{2}^{-}
\end{array}\right)^{-1}, \\
\mathbf{T}_{3}=\left(\begin{array}{cc}
\mathbf{I} & 0 \\
\mathbf{G}_{3}^{+} & 0
\end{array}\right) .
\end{gathered}
$$

Отметим, что обобщение формулы (21) для многопленочной структуры из $N$ слоев можно произвести по индукции, которая приводит к следующим выражениям:

$$
\left(\begin{array}{c}
\hat{E}_{1, x}^{+} \\
\hat{E}_{1, y}^{+} \\
\hat{E}_{1, x}^{-} \\
\hat{E}_{1, y}^{-}
\end{array}\right)=\mathbf{M}\left(\begin{array}{c}
\hat{E}_{N+2, x}^{+} \\
\hat{E}_{N+2, y}^{+} \\
0 \\
0
\end{array}\right)
$$

причем матрица $\mathbf{M}$ имеет вид $\mathbf{M}=\mathbf{T}_{1}\left(\prod_{m=2}^{N+1} \mathbf{T}_{m}\right) \mathbf{T}_{N+2}$, где

$$
\begin{gathered}
\mathbf{T}_{1}=\left(\begin{array}{cc}
\mathbf{I} & \mathbf{I} \\
\mathbf{G}_{1}^{+} & \mathbf{G}_{1}^{-}
\end{array}\right) \\
\mathbf{T}_{m}=\left(\begin{array}{cc}
\mathbf{I} & e^{i k_{m, z} d_{m-1} \mathbf{I}} \\
\mathbf{G}_{m}^{+} & e^{i k_{m, z} d_{m-1}} \mathbf{G}_{m}^{-}
\end{array}\right)\left(\begin{array}{cc}
e^{i k_{m, z} d_{m-1} \mathbf{I}} & \mathbf{I} \\
e^{i k_{m, z} d_{m-1}} \mathbf{G}_{m}^{+} & \mathbf{G}_{m}^{-}
\end{array}\right)^{-1} \\
\mathbf{T}_{N+2}=\left(\begin{array}{cc}
\mathbf{I} & 0 \\
\mathbf{G}_{N+2}^{+} & 0
\end{array}\right) .
\end{gathered}
$$

Отметим, что обычно интересуются задачей падения заданной волны на многопленочную структуру. Тогда эту падающую волну можно разложить в ряд Фурье и найти функции $\hat{E}_{1, x}(\xi, \eta), \hat{E}_{1, y}^{+}(\xi, \eta)$. Разделив матрицу М в (22) на четыре $2 \times 2$-матрицы $\mathbf{M}_{\mathrm{A}}, \mathbf{M}_{\mathrm{B}}, \mathbf{M}_{\mathrm{C}}$ и $\mathbf{M}_{\mathrm{D}}$ :

$$
\mathbf{M}=\left(\begin{array}{ll}
\mathbf{M}_{\mathrm{A}} & \mathbf{M}_{\mathrm{B}} \\
\mathbf{M}_{\mathrm{C}} & \mathbf{M}_{\mathrm{D}}
\end{array}\right)
$$

получим матричные уравнения

$$
\left(\begin{array}{c}
\hat{E}_{1, x}^{+} \\
\hat{E}_{1, y}^{+}
\end{array}\right)=\mathbf{M}_{\mathrm{A}}\left(\begin{array}{c}
\hat{E}_{N+2, x}^{+} \\
\hat{E}_{N+2, y}^{+}
\end{array}\right), \quad\left(\begin{array}{c}
\hat{E}_{1, x}^{-} \\
\hat{E}_{1, y}^{-}
\end{array}\right)=\mathbf{M}_{\mathrm{C}}\left(\begin{array}{c}
\hat{E}_{N+2, x}^{+} \\
\hat{E}_{N+2, y}^{+}
\end{array}\right),
$$

с помощью которых по компонентам падающей волны $\hat{E}_{1, x}^{+}$и $\hat{E}_{1, y}^{+}$можно найти компоненты прошедшей волны:

$$
\left(\begin{array}{c}
\hat{E}_{N+2, x}^{+} \\
\hat{E}_{N+2, y}^{+}
\end{array}\right)=\mathbf{M}_{\mathrm{A}}^{-1}\left(\begin{array}{c}
\hat{E}_{1, x}^{+} \\
\hat{E}_{1, y}^{+}
\end{array}\right)
$$

и отраженной волны:

$$
\left(\begin{array}{c}
\hat{E}_{1, x}^{-} \\
\hat{E}_{1, y}^{-}
\end{array}\right)=\mathbf{M}_{\mathrm{C}} \mathbf{M}_{\mathrm{A}}^{-1}\left(\begin{array}{c}
\hat{E}_{1, x}^{+} \\
\hat{E}_{1, y}^{+}
\end{array}\right)
$$

Тогда из (8) векторы напряженности падающего и отраженного электрических полей будут определяться следующими формулами:

$$
\begin{aligned}
\mathbf{E}_{1}^{\text {in }}(x, y, z)= & \frac{1}{(2 \pi)^{2}} \int_{-\infty}^{+\infty} \int_{-\infty}^{+\infty}\left(\begin{array}{cc}
1 & 0 \\
0 & 1 \\
-\frac{\xi}{k_{1, z}} & -\frac{\eta}{k_{1, z}}
\end{array}\right)\left(\begin{array}{c}
\hat{E}_{1, x}^{+} \\
\hat{E}_{1, y}^{+}
\end{array}\right) \\
& \times e^{i k_{1, z} z} e^{i(\xi x+\eta y)} d \xi d \eta \\
\mathbf{E}_{1}^{\mathrm{ref}}(x, y, z)= & \frac{1}{(2 \pi)^{2}} \int_{-\infty}^{+\infty} \int_{-\infty}^{+\infty}\left(\begin{array}{cc}
1 & 0 \\
0 & 1 \\
\frac{\xi}{k_{1, z}} & \frac{\eta}{k_{1, z}}
\end{array}\right) \\
& \times \mathbf{M}_{\mathrm{C}} \mathbf{M}_{\mathrm{A}}^{-1}\left(\begin{array}{c}
\hat{E}_{1, x}^{+} \\
\hat{E}_{1, y}^{+}
\end{array}\right) e^{-i k_{1, z} z} e^{i(\xi x+\eta y)} d \xi d \eta
\end{aligned}
$$

а напряженность прошедшего электрического поля по аналогии с $(10)$ - формулой

$$
\begin{gathered}
\mathbf{E}_{N+2}{ }^{\operatorname{trans}}(x, y, z)=\frac{1}{(2 \pi)^{2}} \int_{-\infty}^{+\infty} \int_{-\infty}^{+\infty}\left(\begin{array}{cc}
1 & 0 \\
0 & 1 \\
-\frac{\xi}{k_{N+2, z}} & -\frac{\eta}{k_{N+2, z}}
\end{array}\right) \\
\quad \times \mathbf{M}_{\mathrm{A}}^{-1}\left(\begin{array}{c}
\hat{E}_{1, x}^{+} \\
\hat{E}_{1, y}^{+}
\end{array}\right) e^{i k_{N+2, z}\left(z-d_{\mathrm{tot}}\right)} e^{i(\xi x+\eta y)} d \xi d \eta
\end{gathered}
$$

где $d_{\mathrm{tot}}=\sum_{i=1} d_{i}-$ полная толщина многопленочной структуры.

С помощью соотношений (11) и (12) легко можно выписать и выражения для падающего, отраженного и прошедшего магнитных полей (для экономии места мы здесь этого делать не будем). 


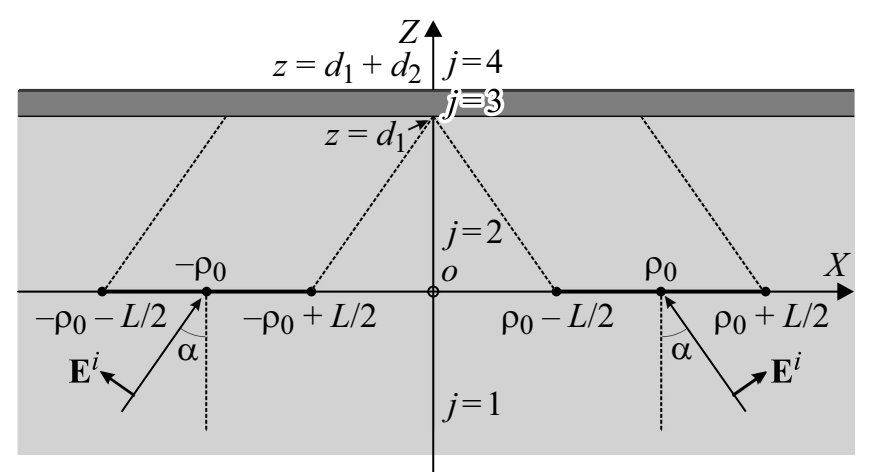

Рис. 3. Геометрия падения конической волны. Область $j=1-$ полупространство призмы; $j=2-$ вспомогательная пленка толщины $d_{1}$ из того же материала, что и призма; $j=3$ - металлическая пленка толщиной $d_{2} ; j=4-$ свободное пространство за металлической пленкой.

\section{Задача падения конической электромагнитной волны ограниченной апертуры на плоскослоистую структуру}

Рассмотрим (рис. 3) падение на многопленочную структуру конической волны $\mathbf{E}^{i}$, которую в плоскости $z=0$ определим функцией

$$
\begin{aligned}
& \mathbf{E}_{1}^{+}(x, y, 0)=e^{i\left(-k_{1}\left(\sqrt{x^{2}+y^{2}}-\rho_{0}\right) \sin \alpha+k_{1} z \cos \alpha\right)} \\
& \quad \times\left(-\frac{x \cos \alpha}{\sqrt{x^{2}+y^{2}}} \mathbf{e}_{x}-\frac{y \cos \alpha}{\sqrt{x^{2}+y^{2}}} \mathbf{e}_{y}-\sin \alpha \mathbf{e}_{z}\right),
\end{aligned}
$$

где $k_{1}=n_{1} \omega / c-$ волновое число в первой среде, $c$ - скорость света в вакууме, $n_{1}=\sqrt{\varepsilon_{1}}-$ показатель преломления в первой среде, $\mathbf{e}_{x}, \mathbf{e}_{y}, \mathbf{e}_{z}-$ единичные векторы в направлениях осей $X, Y$ и $Z$ соответственно, $\alpha-$ угол падения волны на плоскость $z=0$ в локальноплоском приближении. Вектор напряженности электрического поля волны предполагается ориентированным всегда в плоскости падения ( $p$-поляризация). На рис. 3 показана геометрия задачи в разрезе плоскостью $X Z$. Рассматриваемые области задачи мы пронумеруем следующим образом: $j=1-$ полупространство призмы; $j=2-$ вспомогательная пленка из того же материала, что и призма толщины $d_{1}$ (введение этой пленки даст возможность определить закономерности схождения конической падающей волны); $j=3-$ металлическая пленка толщиной $d_{2} ; j=4-$ свободное пространство за металлической пленкой. В такой постановке задачи мы задаем поле падающей конической волны на некотором расстоянии $d_{1}$ от металлической пленки. Отметим, что экспериментальные методы создания таких конических волн с радиальной поляризацией хорошо развиты [17-19].

Пусть при $z=0$ апертура волны ограничена таким образом, чтобы амплитуда волны (26) была постоянной (в локально-плоском приближении амплитуда равна единице) и отлична от нуля только в кольце $\rho \in\left[\rho_{0}-L / 2, \rho_{0}+L / 2\right]$ (где $\rho=\sqrt{x^{2}+y^{2}}$ и $\rho_{0}>L / 2$, причем центральная окружность этого кольца имеет радиус $\left.\rho_{0}\right)$. Тогда компоненты Фурье этой волны при $z=0$ можно зависать в виде

$$
\begin{aligned}
\hat{E}_{1, x}(\xi, \eta)= & -\iint \frac{x \cos \alpha}{\sqrt{x^{2}+y^{2}}} \\
& \times e^{-i k_{1}\left(\sqrt{x^{2}+y^{2}}-\rho_{0}\right) \sin \alpha} e^{-i(\xi x+\eta y)} d x d y, \\
\hat{E}_{1, y}(\xi, \eta)= & -\iint \frac{y \cos \alpha}{\sqrt{x^{2}+y^{2}}} \\
& \times e^{-i k_{1}\left(\sqrt{x^{2}+y^{2}}-\rho_{0}\right) \sin \alpha} e^{-i(\xi x+\eta y)} d x d y .
\end{aligned}
$$

Интегрирование в (27) и (28) производится по указанному кольцу. Перейдем в (27) к полярным координатам $(\rho, \varphi)$, тогда

$$
\begin{aligned}
& \hat{E}_{1, x}^{\mathrm{in}}(\xi, \eta)=-\int_{0}^{2 \pi} \int_{\rho_{0}-L / 2}^{\rho_{0}+L / 2} \frac{\rho \cos \varphi \cos \alpha}{\rho} e^{-i k_{1}\left(\rho-\rho_{0}\right) \sin \alpha} \\
& \times e^{-i(\xi \rho \cos \varphi+\eta \rho \sin \varphi)} \rho d \rho d \varphi=-\cos \alpha e^{i k_{1} \rho_{0} \sin \alpha} \\
& \times \int_{0}^{2 \pi} F\left(-k_{1} \sin \alpha-\xi \cos \varphi-\eta \sin \varphi\right) \cos \varphi d \varphi,
\end{aligned}
$$

где

$$
\begin{aligned}
F(\chi)= & \int_{\rho_{0}-L / 2}^{\rho_{0}+L / 2} e^{i \rho \chi} \rho d \rho=\frac{e^{i \chi \rho_{0}}}{\chi}\left\{2 \rho_{0} \sin \left(\frac{\chi L}{2}\right)\right. \\
& \left.+i\left(\frac{2}{\chi} \sin \left(\frac{\chi L}{2}\right)-L \cos \left(\frac{\chi L}{2}\right)\right)\right\} .
\end{aligned}
$$

Заметим, что

$$
\xi \cos \varphi+\eta \sin \varphi=\sqrt{\xi^{2}+\eta^{2}} \cos (\varphi-\vartheta),
$$

где

$$
\vartheta=\operatorname{arctg}(\eta / \xi) .
$$

Отсюда получаем

$$
\begin{aligned}
-k_{1} \sin \alpha & -\xi \cos \varphi-\eta \sin \varphi \\
= & -k_{1} \sin \alpha-\sqrt{\xi^{2}+\eta^{2}} \cos (\varphi-\vartheta)
\end{aligned}
$$

и выражение (29) примет вид

$$
\begin{aligned}
& \hat{E}_{1, x}^{\mathrm{in}}(\xi, \eta)=-\cos \alpha e^{i k_{1} \rho_{0} \sin \alpha} \\
& \times \int_{0}^{2 \pi} F\left(-k_{1} \sin \alpha-\sqrt{\xi^{2}+\eta^{2}} \cos (\varphi-\vartheta)\right) \cos \varphi d \varphi .
\end{aligned}
$$



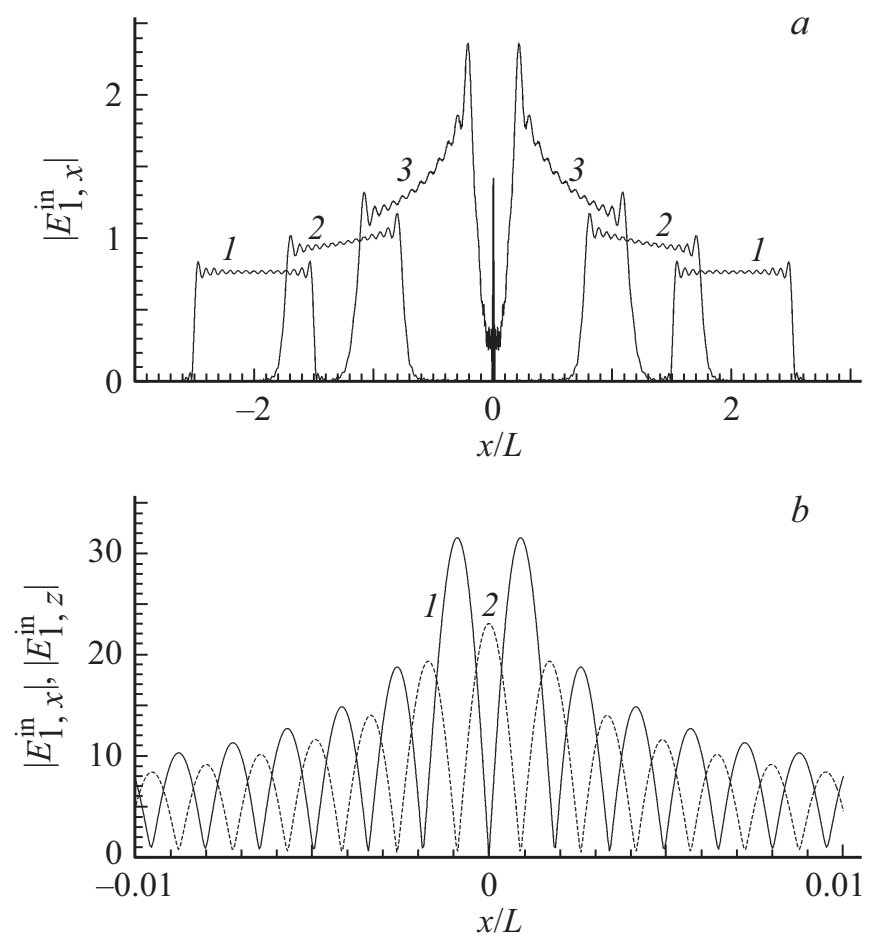

Рис. 4. (a) Результаты расчетов по формуле (23) зависимости модуля $x-$ составляющей падающей волны $\left|E_{1, x}^{\text {in }}(x, 0, z)\right|$ от координаты $x$ для трех фиксированных значений $z: z=0(1)$, $z=0.5 d_{1}(2), z=0.9 d_{1}(3) ;(b)$ зависимости $\left|E_{1, x}^{\text {in }}\left(x, 0, d_{1}\right)\right|$ (сплошная кривая 1$)$ и $\left|E_{1, z}^{\text {in }}\left(x, 0, d_{1}\right)\right|$ (пунктирная кривая 2 ) от $x$ в окрестности $x=0$.

Аналогично из (28) получаем

$$
\begin{aligned}
& \hat{E}_{1, y}^{\text {in }}(\xi, \eta)=-\cos \alpha e^{i k_{1} \rho_{0} \sin \alpha} \\
& \times \int_{0}^{2 \pi} F\left(-k_{1} \sin \alpha-\sqrt{\xi^{2}+\eta^{2}} \cos (\varphi-\vartheta)\right) \sin \varphi d \varphi .
\end{aligned}
$$

Подставляя (30) и (31) в уравнения (24) и (25), можно найти отраженное электрическое поле (24) в первой среде и прошедшее электрическое поле (25) в полупространстве за пленочной структурой.

Итак, рассмотрим сначала вспомогательную задачу распространения без металлической пленки. Рассмотрим вопрос, как сходится коническая волна к плоскости $z=d_{1}=\left(\rho_{0}-L / 2\right) \operatorname{ctg} \alpha$, на которой в дальнейшем будет помещена металлическая пленка. Указанное значение $d_{1}=\left(\rho_{0}-L / 2\right) \operatorname{ctg} \alpha$ выбрано таким образом, чтобы на этом расстоянии крайние внутренние лучи конической волны начали интерферировать на оси системы при $x=0$. Для определенности примем, что $L=200 \mu \mathrm{m}$, $\rho_{0}=2 L$, показатель преломления призмы $n_{1}=1.6$ (т.е. $\left.\varepsilon_{1}=1.6^{2}\right)$, длина падающей волны в вакууме $\lambda=633 \mathrm{~nm}$. Значение угла падения $\alpha=40.041^{\circ}$. Это значение $\alpha$ и геометрия падающей волны соответствуют наилучшему возбуждению поверхностной плазмонной волны на поверхности серебряной пленки [8].
На рис. 4, а показаны результаты расчетов по формуле (23) зависимости модуля $x$-компоненты падающей волны $\left|E_{1, x}^{\text {in }}(x, 0, z)\right|$ от $x$ для трех фиксированных значений $z: a=0, z=0.5 d_{1}, z=0.9 d_{1}$. Из полученных зависимостей видно, что амплитуда конической волны увеличивается по мере ее схождения к оси $Z$. Это ясно из геометрических соображений. Кроме того, видно, что на кривой 3 (при $z=0.9 d_{1}$ ) вблизи $x=0$ наблюдается интерференция на расстояниях порядка длины волны, которая не разрешается на данном рисунке. Как показали расчеты, при $z=d_{1}$ (геометрическом наложении сходящихся волн) данная интерференция еще более усиливается из-за увеличения амплитуд интерферирующих волн.

Детали интерференции для $z=d_{1}$ показаны на рис. $4, b$, на котором представлены зависимости $\left|E_{1, x}^{\text {in }}\left(x, 0, d_{1}\right)\right|$ и $\left|E_{1, z}^{\text {in }}\left(x, 0, d_{1}\right)\right|$ в окрестности $x=0$. Из полученных зависимостей видно, что, конечно, коническая волна не фокусируется в строгом смысле этого слова. В окрестности $x=0$ получаются интерференционные кольца с близкой амплитудой в максимумах.

Итак, после рассмотрения вспомогательной задачи падения конической волны на пленку исследуем далее поле поверхностной волны на свободной границе пленки.

\section{Вычисление сфокусированного поля на свободной границе пленки и обсуждение полученных результатов}

Рассмотрим возбуждение конической волной (26) поверхностных плазмонных волн на свободной поверхности металлической пленки многопленочной структуры, показанной на рис. 3. Предполагается, что пленка 3 серебряная, а длина падающей волны в вакууме, как и ранее, равна $633 \mathrm{~nm}$. Параметры сред, показанных на рис. 3, на рабочей длине волны представлены в таблице.

Было вычислено распределение электрического поля на свободной поверхности металлической пленки

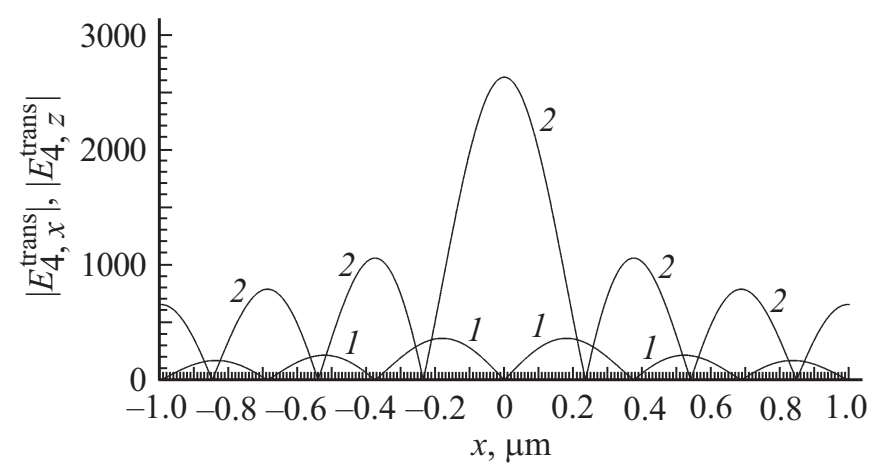

Рис. 5. Интерференционная картина фокусировки радиально сходящейся поверхностной плазмонной волны на свободной границе серебряной пленки: $\left|E_{4, x}^{\text {trans }}\left(x, 0, d_{1}+d_{2}\right)\right|(1)$ и $\left|E_{4, z}\left(x, 0, d_{1}+d_{2}\right)\right|(2)$. Величины нормированы на амплитуду падающей волны в призме при $z=0$. 


\begin{tabular}{c|c|c|c}
\hline $\begin{array}{c}\text { Порядковый } \\
\text { номер среды, } j\end{array}$ & Название слоя & $\begin{array}{c}\text { Относительная } \\
\text { диэлектрическая проницаемость }\end{array}$ & Толщина пленки \\
\hline 1 & Призма & $1.6^{2}$ & Полубесконечная среда \\
2 & Слой материала призмы & $1.6^{2}$ & $d_{1}=\left(\rho_{0}-L / 2\right) \operatorname{ctg} \alpha=357 \mu \mathrm{m}$ \\
3 & Серебряная пленка & $\varepsilon_{m}=-18.2+i 0.5$ & $\mathrm{~nm}$ \\
4 & Свободное пространство (воздух) & 1 & Полубесконечная среда
\end{tabular}

$\left(z=d_{\mathrm{tot}}=d_{1}+d_{2}\right)$ по формуле $(25)$. Результаты распределения модулей $x$ - и $z$-составляющих напряженности электрического поля в области фокуса в плоскости $X Z$ показаны на рис. 5. Электрическое поле симметрично относительно поворотов вокруг оси $Z$. В области фокуса $(x, y, z)=\left(0,0, d_{1}+d_{2}\right)$ на поверхности пленки наблюдается максимум поверхностной волны, причем $z$-составляющая напряженности поля заметно выше радиальных составляющих. Особо следует отметить большую величину поля в максимуме фокуса, 2633 в единицах амплитуды падающей в призме волны в плоскости $z=0$. Даже если сравнивать это значение с максимальным значением $z$-составляющей напряженности падающего электрического поля на границе пленки и призмы, т.е. $\left|E_{1, z}^{\text {in }}\left(0,0, d_{1}\right)\right|$, то поле усиливается более чем на два порядка. Такое большое усиление поля определяется следующими причинами:

- ширина падающей волны $L=200 \mu \mathrm{m}$ в направлении, перпендикулярном оси, выбиралась достаточно большой - чтобы амплитуда поверхностной волны достигала значения, близкого к максимальному значению [8];

- угол падения $\alpha=40.041^{\circ}$ соответствует оптимальному углу возбуждения поверхностной волны в плоской задаче с ограниченным падающим пучком [8];

- точка схождения внутренних лучей падающей волны к внутренней поверхности пленки $(x, y, z)=\left(0,0, d_{1}\right) \quad$ соответствует максимальному полю поверхностной волны на другой стороне пленки, исходя из результатов решения плоской задачи [8];

- электрическое поле падающей волны поляризовано в плоскости падения (в плоскости, проходящей через ось задачи $Z$ ), что обеспечивает возбуждение только радиально сходящейся поверхностной волны с той же симметрией.

Расчеты показали, что если менять расстояние $d_{1}$, то максимальное значение напряженности поля в фокусе на свободной поверхности пленки слабо меняется при изменении $d_{1}$ в окрестности значения $\left(\rho_{0}-L / 2\right) \operatorname{ctg} \alpha$. Практически здесь фокальное поле равно максимальному значению. Поэтому настройка на фокальный максимум полученной системы не должна представлять проблемы.

\section{Заключение}

Предложен теоретический метод исследования дифракционных явлений при фокусировке радиально сходящейся поверхностной плазмонной волны. Найдены условия оптимальной фокусировки при возбуждении системы конической волной с $p$-поляризацией. Найдены фокальные распределения поля на свободной поверхности металлической пленки и величины усиления электрического поля в фокусе.

Полученный результат имеет прикладное значение в таких приложениях, как создание локальной оптической эмиссии электронов с поверхности металлических пленок [20,21], локальной генерации высших оптических гармоник [22]. Полученные результаты помогут оптимизировать устройства по возбуждению на металлическом острие поверхностной плазмонной ТМ-волны, сходящейся к его вершине и создающей на ней гигантское усиление поля [23].

\section{Список литературы}

[1] De Angelis F., Das G., Candeloro P. et al. // Nature Nanotech. 2010. V. 5. P. 67.

[2] Giugni A., Torre B., Toma A. et al. // Nature Nanotech. 2013. V. 8. P. 845.

[3] Giugni A., Allione M., Torre B. et al. // J. Opt. 2014. V. 16. P. 114003.

[4] Rui G., Chen W., Lu Y. et al. // J. Opt. 2010. V. 12. P. 035004.

[5] López-Tejeira F., Rodrigo S.G., Martín-Moreno L. et al. // New J. Phys. 2008. V. 10. P. 033035.

[6] De Angelis F., Patrini M., Das G. et al. // Nano Lett. 2008. V. 8. P. 2321.

[7] Lezec H.J., Thio T. // Opt. Express. 2004. V. 12. N 16. P. 3629.

[8] Петрин А.Б. // Опт. и спектр. 2018. Т. 125. № 6. С. 830.

[9] Piliarik M., Homola J. // Opt. Express. 2009. V. 17. N 19. P. 16505.

[10] Liedberg B., Nylander C., Lundstrom I. // Sensors and Actuators. 1983. V. 4. P. 299.

[11] Liedberg B., Nylander C., Lundstrom I. // Biosensors \& Bioelectronics. 1995. V. 10. P. i-ix.

[12] Garabedian R., Gonzalez C., Richards J. et al. // Sensors and Achrators A. 1994. V. 43. P. 202.

[13] Петрин А.Б. // Опт. и спектр. 2018. Т. 125. № 3. С. 375.

[14] Sommerfeld A. // Annalen der Physik. 1909. V. 333. P. 665.

[15] Бреховских Л.М. Волны в слоистых средах. М.: Наука, 1973. 
[16] Новотный Л., Хехт Б. Основы нанооптики. Пер. с англ. Под ред. Самарцева В.В. М.: Физматлит, 2009.

[17] Налимов А.Г., О'Фаолейн Л., Стафеев С.С., Шанина М.И., Котляр В.В. // Компьютерная оптика. 2014. Т. 38. № 2. С. 229.

[18] Стафеев С.С., Котляр В.В. // Компьютерная оптика. 2017. T. 41. № 2. C. 147.

[19] Ma Y. // Optical Review. 2012. V. 19. N 2. P. 39.

[20] Racz P., Irvine S.E., Lenner M. et al. // Appl. Phys. Lett. 2011. V. 98. N 11. P. 111116.

[21] Welsh G.H., Wynne K. // Opt. Express. 2009. V. 17. P. 2470.

[22] Kim S., Jin J., Kim Y. et al. // Nature. 2008. V. 453. N 5. P. 757.

[23] Giugni A., Allione M., Torre B. et al. // J. Opt. 2014. V. 16. P. 114003. 